\title{
RECURRENCE RELATIONS FOR DISCRETE HYPERGEOMETRIC FUNCTIONS
}

\author{
R. ÁLVAREZ-NODARSE AND J. L. CARDOSO
}

\begin{abstract}
We present a general procedure for finding linear recurrence relations for the solutions of the second order difference equation of hypergeometric type. Applications to wave functions of certain discrete system are also given.
\end{abstract}

\section{INTRODUCTION}

In the last years there has been increasing interest in discrete models in classical and quantum physics (for a recent review see [20]). Several of such models are solved using the theory of the classical discrete polynomials [22]. Important instances of such systems are the discrete oscillators of Charlier [5], Kravchuk oscillators [6, 8, 10, 12, 14] and Meixner oscillators [5] that are related to the polynomials of Charlier, Kravchuk and Meixner, respectively, and the finite radial oscillator $[9,11]$ related with the Hahn polynomials. For applications it is important to have recurrence relations for the discrete wave function of such systems. Methods for obtaining such recurrence relations have attracted the interest of several authors (see e.g. $[19,20]$ and references therein).

Our main aim in this paper is to present a constructive approach for generating recurrence relations and ladder-type operators for some discrete system such as the discrete oscillators $[2,5,6,8,9,10,11,12,13,14]$, discrete Calogero-Sutherland model [20], etc. The main idea is to use the connection of the wave functions with the classical discrete polynomials in a similar way as it was done in our previous paper [16] for the $N$-th dimensional oscillators and hydrogenlike atoms. This approach allows us to recover the relations obtained in $[5,19,20]$ and also to obtain several new relations for the discrete polynomials and therefore for the associated (wave) functions in a constructive way. This can be extended to other exactly solvable models which involve discrete hypergeometric functions or polynomials.

The structure of the paper is as follows: In section 2 the required results and notation from special function theory are introduced. The main results of the paper are in Section 3, where some general existence theorems are stated and proved. In Section 4 simple examples of recurrences and laddertype relations of some discrete systems are presented. Finally, at the end of

Key words and phrases. hypergeometric functions, difference equations, recurrence relations, discrete polynomials, discrete wave functions. 
section 4, we include some more complicate examples, in order to show the interest as well as the power of the method for finding needed recurrence relations. In this way we show how this method can be useful for finding recurrences à la carte, that it seem to be, in general, a very important tool for computations with discrete systems.

\section{The discrete INGREDIENTS}

2.1. "Discrete" preliminaries. Here we collect the basic background [1, $22]$ on hypergeometric discrete polynomials needed in the rest of the work.

Let us consider the second-order difference equation of hypergeometrictype

$$
\sigma(s) \nabla \Delta y(s)+\tau(s) \Delta y(s)+\lambda y(s)=0,
$$

where $\sigma(s)$ and $\tau(s)$ are polynomials of degree not greater than 2 and 1 , respectively, $\lambda$ is a constant, and $\Delta f(s)=f(s+1)-f(s)$ and $\nabla f(s)=$ $\Delta f(s-1)$ are the forward and backward difference operators, respectively. This equation can be written in self-adjoint form

$$
\Delta[\sigma(s) \rho(s) \nabla y(s)]+\lambda \rho(s) y(s)=0,
$$

where the function $\rho(s)$ satisfies the Pearson-type difference equation

$$
\Delta[\sigma(s) \rho(s)]=\tau(s) \rho(s) .
$$

For the solutions of the difference equation (2.1) the following theorem holds

Theorem 2.1. [23, page 136] The difference equation (2.1) has particular solutions of the form

$$
y_{\nu}(s)=\frac{C_{\nu}}{\rho(s)} \sum_{x=a}^{b-1} \frac{\rho_{\nu}(x)}{(x-s)_{\nu+1}}
$$

if the condition

$$
\left.\frac{\sigma(x) \rho_{\nu}(x)}{(x-s-1)_{\nu+2}}\right|_{a} ^{b}=0
$$

is satisfied, and has solutions of the form

$$
y_{\nu}(s)=\frac{C_{\nu}}{\rho(s)} \int_{C} \frac{\rho_{\nu}(x) d x}{(x-s)_{\nu+1}}
$$

if the condition

$$
\int_{C} \frac{\sigma(x+1) \rho(x+1) d x}{(x-s)_{\nu+2}}=\int_{C} \frac{\sigma(x) \rho(x) d x}{(x-s-1)_{\nu+2}}
$$

is satisfied. Here $C$ is a contour in the complex plane, $C_{\nu}$ is a constant, $\rho(s)$ and $\rho_{\nu}(s)$ are the solution of the Pearson type equations

$$
\Delta[\sigma(s) \rho(s)]=\tau(s) \rho(s), \quad \Delta\left[\sigma(s) \rho_{\nu}(s)\right]=\tau(s) \rho_{\nu}(s),
$$


where $\tau_{\nu}(s)=\sigma(s+\nu)-\sigma(s)+\tau(s+\nu)$, $\nu$ is the root of the equation $\lambda+\nu \tau^{\prime}+1 / 2 \nu(\nu-1) \sigma^{\prime \prime}=0$, and $(x)_{\nu}$ denotes the Pochhammer symbols or shifted factorials

$$
(x)_{\nu}:=\frac{\Gamma(x+\nu)}{\Gamma(x)} .
$$

Important instances of the functions $y_{\nu}$ are the classical discrete polynomials which are given by [23, page 139]

$$
P_{n}(s)=\frac{n ! B_{n}}{\rho(s) 2 \pi i} \int_{C} \frac{\rho_{n}(x)}{(x-s)_{n+1}} d x,
$$

when $C$ is a closed contour surrounding the points $x=s, s-1, \ldots, s-n$ and it is assumed that $\rho_{n}(x)$ and $\rho_{n}(x+1)$ are analytic inside $C$, i.e., they correspond to formula (2.5). In this case

$$
\lambda:=\lambda_{n}=-n \Delta \tau(s)-\frac{1}{2} n(n-1) \sigma^{\prime \prime}, \quad n=0,1,2, \ldots .
$$

2.2. The classical discrete polynomials. The classical discrete orthogonal polynomials are orthogonal on the integers in $[a, b-1]$ with respect to the weight function $\rho(s)$, i.e.,

$$
\sum_{x=a}^{b-1} P_{n}(s) P_{m}(s) \rho(s)=\delta_{n m} d_{n}^{2},
$$

provided that the boundary condition $\left.\sigma(s) \rho(s) x^{k}\right|_{x=a, b}=0$, for all $k \geq 0$, holds, where $d_{n}^{2}$ is the square of the norm of the polynomial $P_{n}(s)$. They can be obtained using the so-called Rodrigues-type formula

$$
P_{n}(s)=\frac{B_{n}}{\rho(s)} \nabla^{n}\left[\rho_{n}(s)\right], \quad n=0,1,2, \ldots, .
$$

where $B_{n}$ is the normalization constant and

$$
\rho_{n}(s)=\rho(s+n) \prod_{m=1}^{n} \sigma(s+m) .
$$

Furthermore, for the $k$-th differences we have [23, Eq. 20, page 110]

$$
\Delta^{k} P_{n}(s)=\frac{A_{n k} B_{n}}{\rho_{k}(s)} \nabla^{n-k}\left[\rho_{n}(s)\right],
$$

where

$$
A_{n k}=\frac{n !}{(n-k) !} \prod_{m=0}^{k-1}\left[\tau^{\prime}+(n+m-1) \frac{\sigma^{\prime \prime}}{2}\right] .
$$

A simple consequence of the orthogonality is the three-term recurrence relation (TTRR) that the polynomials $P_{n}$ satisfy

$$
x P_{n}(s)=\alpha_{n} P_{n+1}(s)+\beta_{n} P_{n}(s)+\gamma_{n} P_{n-1}(s) .
$$

Also they satisfy several difference-recurrence relations [1] 


$$
\begin{gathered}
\sigma(s) \nabla P_{n}(s)=\frac{\lambda_{n}}{n \tau_{n}^{\prime}}\left[\tau_{n}(s) P_{n}(s)-\frac{B_{n}}{B_{n+1}} P_{n+1}(s)\right] \\
{[\sigma(s)+\tau(s)] \Delta P_{n}(s)=\frac{\lambda_{n}}{n \tau_{n}^{\prime}}\left\{\left[\tau_{n}(s)-n \tau_{n}^{\prime}\right] P_{n}(s)-\frac{B_{n}}{B_{n+1}} P_{n+1}(s)\right\}} \\
\sigma(s) \nabla P_{n}(s)=\widetilde{\alpha}_{n} P_{n+1}(s)+\widetilde{\beta}_{n} P_{n}(s)+\widetilde{\gamma}_{n} P_{n-1}(s) \\
{[\sigma(s)+\tau(s)] \Delta P_{n}(s)=\widehat{\alpha}_{n} P_{n+1}(s)+\widehat{\beta}_{n} P_{n}(s)+\widehat{\gamma}_{n} P_{n-1}(s)} \\
P_{n}(s)=Q_{n}(s)+\delta_{n} Q_{n-1}(s)+\epsilon_{n} Q_{n-2}(s)
\end{gathered}
$$

where $Q_{n}(s)=\Delta P_{n+1}(s) /(n+1)$.

\subsection{Classical families of Hahn, Meixner, Kravchuk and Charlier.}

The four families of classical discrete orthogonal polynomials are the Hahn

\begin{tabular}{|c|c|c|c|c|}
\hline$P_{n}$ & $\begin{array}{c}\text { Hahn } \\
h_{n}^{\alpha, \beta}(s ; N)\end{array}$ & $\begin{array}{l}\text { Meixner } \\
M_{n}^{\gamma, \mu}(s)\end{array}$ & $\begin{array}{c}\text { Kravchuk } \\
K_{n}^{p}(s)\end{array}$ & $\begin{array}{c}\text { Charlier } \\
C_{n}^{\mu}(s)\end{array}$ \\
\hline$[a, b]$ & {$[0, N]$} & {$[0, \infty)$} & {$[0, N+1]$} & {$[0, \infty)$} \\
\hline$\sigma$ & $s(N+\alpha-s)$ & $s$ & $s$ & $s$ \\
\hline$\tau$ & $(\beta+1)(N-1)-(\alpha+\beta+2) s$ & $(\mu-1) s+\mu \gamma$ & $\frac{N p-s}{1-p}$ & $\mu-s$ \\
\hline$\tau_{n}$ & $\begin{array}{c}(\beta+1)(N-1)+n(N-\beta-n-2) \\
-(\alpha+\beta+2 n+2) s\end{array}$ & $(\mu-1) s+\mu(\gamma+n)$ & $\frac{(N-n) p-s}{1-p}$ & $\mu-s$ \\
\hline$\sigma+\tau$ & $(s+\beta+1)(N-1-s)$ & $\mu s+\gamma \mu$ & $-\frac{p}{1-p}(s-N)$ & $\mu$ \\
\hline$\lambda_{n}$ & $n(n+\alpha+\beta+1)$ & $(1-\mu) n$ & $\frac{n}{1-p}$ & $n$ \\
\hline$\rho$ & $\begin{array}{c}\frac{\Gamma(N+\alpha-s) \Gamma(\beta+s+1)}{\Gamma(N-s) \Gamma(s+1)} \\
\alpha, \beta \geq-1, n \leq N-1\end{array}$ & $\begin{array}{c}\frac{\mu^{s} \Gamma(\gamma+s)}{\Gamma(\gamma) \Gamma(s+1)} \\
\gamma>0,0<\mu<1\end{array}$ & $\begin{array}{l}\frac{N ! p^{s}(1-p)^{N-s}}{\Gamma(N+1-s) \Gamma(s+1)} \\
0<p<1, n \leq N-1\end{array}$ & $\begin{array}{c}\frac{e^{-\mu} \mu^{s}}{\Gamma(s+1)} \\
\mu>0\end{array}$ \\
\hline$\rho_{n}$ & $\frac{\Gamma(N+\alpha-s) \Gamma(n+\beta+s+1)}{\Gamma(N-n-s) \Gamma(s+1)}$ & $\frac{\mu^{s+n} \Gamma(\gamma+n+s)}{\Gamma(\gamma) \Gamma(s+1)}$ & $\frac{N ! p^{s+n}(1-p)^{N-n-s}}{\Gamma(N+1-n-s) \Gamma(s+1)}$ & $\frac{e^{-\mu} \mu^{s+n}}{\Gamma(s+1)}$ \\
\hline
\end{tabular}
$h_{n}^{\alpha, \beta}(x, N)$, Meixner $M_{n}^{\gamma, \mu}(s)$, Kravchuk $K_{n}^{p}(x, N)$ and Charlier $C_{n}^{\mu}(s)$, polynomials $[18,22,23]$, whose main data in its monic form are shown in Tables 1,2 and 3.

TABLE 1. Classification of discrete classical polynomials

They can be expressed in terms of hypergeometric functions by [22, Section 2.7,p. 49]:

$$
\begin{gathered}
h_{n}^{\alpha, \beta}(x, N)=\frac{(1-N)_{n}(\beta+1)_{n}}{(\alpha+\beta+n+1)_{n}} \mathrm{~F}_{2}\left(\begin{array}{c}
-x, \alpha+\beta+n+1,-n \\
1-N, \beta+1
\end{array} \mid 1\right), \\
M_{n}^{\gamma, \mu}(s)=(\gamma)_{n} \frac{\mu^{n}}{(\mu-1)^{n}}{ }_{2} \mathrm{~F}_{1}\left(\begin{array}{c}
-n,-x \\
\gamma
\end{array} \mid 1-\frac{1}{\mu}\right),
\end{gathered}
$$


TABLE 2. Main data for monic Hahn and Chebyshev polynomials

\begin{tabular}{|c|c|c|}
\hline & $\begin{array}{c}\text { Hahn } \\
h_{n}^{\alpha, \beta}(s ; N)\end{array}$ & $\begin{array}{c}\text { Chebyshev } \\
t_{n}(s ; N)=h_{n}^{0,0}(s ; N)\end{array}$ \\
\hline$B_{n}$ & $\frac{(-1)^{n}}{(\alpha+\beta+n+1)_{n}}$ & $\frac{(-1)^{n}}{(n+1)_{n}}$ \\
\hline$b_{n}$ & $-\frac{n}{2}\left(\frac{2(\beta+1)(N-1)+(n-1)(\alpha-\beta+2 N-2)}{\alpha+\beta+2 n}\right)$ & $-\frac{n(N-1)}{2}$ \\
\hline$d_{n}^{2}$ & $\frac{n ! \Gamma(\alpha+n+1) \Gamma(\beta+n+1) \Gamma(\alpha+\beta+N+n+1)}{(\alpha+\beta+2 n+1)(N-n-1) ! \Gamma(\alpha+\beta+n+1)(\alpha+\beta+n+1)_{n}^{2}}$ & $\frac{n !^{2}(N+n) !(n+1)_{n}^{-2}}{(2 n+1)(N-n-1) !}$ \\
\hline$\beta_{n}$ & $\begin{array}{c}\frac{(\beta+1)(N-1)(\alpha+\beta)+n(2 N+\alpha-\beta-2)(\alpha+\beta+n+1)}{(\alpha+\beta+2 n)(\alpha+\beta+2 n+2)} \\
\frac{n(N-n)(\alpha+\beta+n)(\alpha+n)(\beta+n)(\alpha+\beta+N+n)}{(\alpha+\beta+2 n-1)(\alpha+\beta+2 n)^{2}(\alpha+\beta+2 n+1)}\end{array}$ & $\begin{array}{l}\frac{N-1}{2} \\
\frac{n^{2}\left(N^{2}-n^{2}\right)}{4(2 n-1)(2 n+1)}\end{array}$ \\
\hline $\begin{array}{l}\widehat{\alpha}_{n} \\
\widehat{\beta}_{n}\end{array}$ & $\begin{array}{c}-n \\
\frac{n\left(-\alpha-\beta-\alpha \beta-\beta^{2}-2 n-2 \alpha n-2 \beta n-2 n^{2}+\alpha N-\beta N\right)}{(\alpha+\beta+n+1)^{-1}(\alpha+\beta+2 n)(\alpha+\beta+2 n+2)} \\
-\frac{n(\alpha+\beta+n)(\alpha+\beta+n+1)(\alpha+n)(\beta+n)(\alpha+\beta+N+n)}{(n-N)^{-1}(\alpha+\beta+2 n-1)(\alpha+\beta+2 n)^{2}(\alpha+\beta+2 n+1)}\end{array}$ & $\begin{array}{c}-n \\
-\frac{n(n+1)}{2} \\
-\frac{n^{2}(n+1)\left(N^{2}-n^{2}\right)}{4(2 n-1)(2 n+1)} \\
\end{array}$ \\
\hline $\begin{array}{l}\widetilde{\alpha}_{n} \\
\widetilde{\beta}_{n}\end{array}$ & $\begin{array}{c}-n \\
\frac{n\left(\alpha+\alpha^{2}+\beta+\alpha \beta+2 n+2 \alpha n+2 \beta n+2 n^{2}+\alpha N-\beta N\right)}{(\alpha+\beta+n+1)^{-1}(\alpha+\beta+2 n)(\alpha+\beta+2 n+2)} \\
-\frac{n(\alpha+\beta+n)(\alpha+\beta+n+1)(\alpha+n)(\beta+n)(\alpha+\beta+N+n)}{(n-N)^{-1}(\alpha+\beta+2 n-1)(\alpha+\beta+2 n)^{2}(\alpha+\beta+2 n+1)}\end{array}$ & $\begin{array}{c}-n \\
\frac{n(n+1)}{2} \\
-\frac{n^{2}(n+1)\left(N^{2}-n^{2}\right)}{4(2 n-1)(2 n+1)}\end{array}$ \\
\hline$\delta_{n}$ & $\begin{array}{c}\frac{n(\alpha-\beta)(2 N+\alpha+\beta)}{2(\alpha+\beta+2 n)(\alpha+\beta+2 n+2)}-\frac{n}{2} \\
\frac{n(n-1)(N-n)(\alpha+n)(\beta+n)(\alpha+\beta+N+n)}{(\alpha+\beta+2 n-1)(\alpha+\beta+2 n)^{2}(\alpha+\beta+2 n+1)}\end{array}$ & $\begin{array}{c}-\frac{n}{2} \\
-\frac{n(n-1)\left(N^{2}-n^{2}\right)}{4(2 n-1)(2 n+1)}\end{array}$ \\
\hline & $K_{n}^{p}(x, N)=\frac{(-p)^{n} N !}{(N-n) !} \quad{ }_{2} \mathrm{~F}_{1}\left(\begin{array}{c}-n,-x \\
-N\end{array}\right.$ & \\
\hline
\end{tabular}

where the generalized hypergeometric function ${ }_{p} \mathrm{~F}_{q}$ is defined by

$$
{ }_{p} \mathrm{~F}_{q}\left(\begin{array}{c}
a_{1}, a_{2}, \ldots, a_{p} \\
b_{1}, b_{2}, \ldots, b_{q}
\end{array} \mid x\right)=\sum_{k=0}^{\infty} \frac{\left(a_{1}\right)_{k}\left(a_{2}\right)_{k} \cdots\left(a_{p}\right)_{k}}{\left(b_{1}\right)_{k}\left(b_{2}\right)_{k} \cdots\left(b_{q}\right)_{k}} \frac{x^{k}}{k !} .
$$

A very important special case of the Hahn polynomials $(\alpha=\beta=0)$ are the discrete Chebyshev polynomials $t_{n}(x, N):=h_{n}^{0,0}(x, N)$.

\section{General Recurrence Relations}

In this section we will obtain several recurrence relations for the solutions (2.3) and (2.5) of the difference equation (2.1). We start with the following lemma that is the discrete analog of Lemma in [23, page 14]. 
TABle 3. Main data for monic Charlier, Meixner and Kravchuk polynomials

\begin{tabular}{|c|c|c|c|}
\hline & $\begin{array}{c}\text { Charlier } \\
C_{n}^{\mu}(s)\end{array}$ & $\begin{array}{c}\text { Kravchuk } \\
K_{n}^{p}(s)\end{array}$ & $\begin{array}{l}\text { Meixner } \\
M_{n}^{\gamma, \mu}(s)\end{array}$ \\
\hline$B_{n}$ & $(-1)^{n}$ & $(-1)^{n}(1-p)^{n}$ & $\frac{1}{(\mu-1)^{n}}$ \\
\hline$b_{n}$ & $-\frac{n}{2}(2 \mu+n-1)$ & $-n[N p+(n-1)(1 / 2-p)]$ & $\left(\frac{n \mu}{\mu-1}\right)\left(\gamma+\frac{n-1}{2} \frac{\mu+1}{\mu}\right)$ \\
\hline$d_{n}^{2}$ & $n ! \mu^{n}$ & $\frac{n ! N ! p^{n}(1-p)^{n}}{(N-n) !}$ & $\frac{n !(\gamma)_{n} \mu^{n}}{(1-\mu)^{\gamma+2 n}}$ \\
\hline$\beta_{n}$ & $\begin{array}{c}n+\mu \\
n \mu\end{array}$ & $\begin{array}{c}N p+(1-2 p) n \\
n p(1-p)(N-n+1)\end{array}$ & $\begin{array}{l}\frac{n(1+\mu)+\mu \gamma}{1-\mu} \\
\frac{n \mu(n-1+\gamma)}{(\mu-1)^{2}}\end{array}$ \\
\hline $\begin{array}{l}\widehat{\alpha}_{n} \\
\widehat{\beta}_{n} \\
\widehat{\gamma}_{n}\end{array}$ & $\begin{array}{c}0 \\
0 \\
n \mu\end{array}$ & $\begin{array}{c}0 \\
-\frac{n p}{1-p} \\
p n(N-n+1)\end{array}$ & $\begin{array}{c}0 \\
n \mu \\
\frac{n \mu(n-1+\gamma)}{1-\mu}\end{array}$ \\
\hline $\begin{array}{l}\widetilde{\alpha}_{n} \\
\widetilde{\beta}_{n} \\
\widetilde{\gamma}_{n}\end{array}$ & $\begin{array}{c}0 \\
n \\
n \mu\end{array}$ & $\begin{array}{c}0 \\
n \\
p n(N-n+1)\end{array}$ & $\begin{array}{c}0 \\
n \\
\frac{n \mu(n-1+\gamma)}{1-\mu}\end{array}$ \\
\hline $\begin{array}{l}\delta_{n} \\
\epsilon_{n}\end{array}$ & $\begin{array}{l}0 \\
0\end{array}$ & $\begin{array}{c}n(1-p) \\
0\end{array}$ & $\frac{n \mu}{1-\mu}$ \\
\hline
\end{tabular}

Let us define the functions

$$
\Phi_{\nu \mu}(z)=\sum_{s=a}^{b-1} \frac{\rho_{\nu}(s)}{(s-z)_{\mu+1}}
$$

and

$$
\Phi_{\nu \mu}(z)=\int_{C} \frac{\rho_{\nu}(s) d s}{(s-z)_{\mu+1}}
$$

corresponding to the functions ${ }^{1}(2.3)$ and $(2.5)$, respectively. In fact, the functions $y_{\nu}$ and the functions $\Phi_{\nu \mu}$ are related by the formula

$$
y_{\nu}(z)=\frac{C_{\nu}}{\rho(z)} \Phi_{\nu \nu}(z) \text {. }
$$

Lemma 3.1. Any three functions $\Phi_{\nu_{i} \mu_{i}}, i=1,2,3$ are connected by a linear relation

$$
\sum_{i=1}^{3} A_{i}(z) \Phi_{\nu_{i} \mu_{i}}(z)=0
$$

\footnotetext{
${ }^{1}$ Obviously the functions (3.1) correspond to the boundary condition (2.4), whereas the functions (3.2) correspond to the condition (2.6).
} 
with polynomial coefficients $A_{i}(z)$ which are not all three vanishing provided that the differences $\nu_{i}-\nu_{j}$ and $\mu_{i}-\mu_{j}, i, j=1,2,3$ are integers and that the following condition holds ${ }^{2}$

$$
\left.\frac{s^{k} \sigma(s) \rho_{\nu_{0}}(s)}{(s-z)_{\mu_{0}}}\right|_{s=a} ^{s=b}=0, \quad k=0,1,2, \ldots,
$$

for the case when $\Phi_{\nu_{i} \mu_{i}}$ are given by (3.1) and

$$
\int_{C} \Delta_{s} \frac{s^{k} \sigma(s) \rho_{\nu_{0}}(s) d s}{(s-z)_{\mu_{0}}}=0, \quad k=0,1,2, \ldots,
$$

for the case when $\Phi_{\nu_{i} \mu_{i}}$ are given by (3.2). Here $\nu_{0}$ is the $\nu_{i}, i=1,2,3$ with the smallest real part and $\mu_{0}$ is the $\mu_{i}, i=1,2,3$ with the largest real part.

Proof. We will give the proof for the case of functions of the form (3.1), the other case is completely similar. We have

$$
\begin{aligned}
\sum_{i=1}^{3} A_{i}(z) \Phi_{\nu_{i} \mu_{i}}(z) & =\sum_{i=1}^{3} A_{i}(z) \sum_{s=a}^{b-1} \frac{\rho_{\nu_{i}}(s)}{(s-z)_{\mu_{i}+1}}=\sum_{s=a}^{b-1} \sum_{i=1}^{3} A_{i}(z) \frac{\rho_{\nu_{i}}(s)}{(s-z)_{\mu_{i}+1}} \\
& =\sum_{s=a}^{b-1} \frac{1}{(s-z)_{\mu_{0}+1}}\left(\sum_{i=1}^{3} A_{i} \rho_{\nu_{i}}(s) \frac{(s-z)_{\mu_{0}+1}}{(s-z)_{\mu_{i}+1}}\right) \\
& =\sum_{s=a}^{b-1} \frac{1}{(s-z)_{\mu_{0}+1}}\left(\sum_{i=1}^{3} A_{i} \rho_{\nu_{i}}(s)\left(s-z+\mu_{i}+1\right)_{\mu_{0}-\mu_{i}}\right),
\end{aligned}
$$

where the identity

$$
\frac{(s-z)_{\alpha}}{(s-z)_{\beta}}=(s-z+\beta)_{\alpha-\beta}, \quad \alpha \geq \beta
$$

is used. Next we use the identity $\rho_{\nu}(s)=\sigma(s+1) \rho_{\nu-1}(s+1)$ as well as the Pearson-type equation (2.7) rewritten in the equivalent form

$$
\frac{\rho_{\nu}(s+1)}{\rho_{\nu}(s)}=\frac{\sigma(s)+\tau_{\nu}(s)}{\sigma(s+1)}=\frac{\sigma(s+\nu)+\tau(s+\nu)}{\sigma(s+1)},
$$

which leads to

$$
\frac{\rho\left(s+\nu_{i}\right)}{\rho\left(s+\nu_{0}\right)}=\frac{\sigma\left(s+\nu_{i}-1\right)+\tau\left(s+\nu_{i}-1\right)}{\sigma\left(s+\nu_{i}\right)} \cdots \frac{\sigma\left(s+\nu_{0}\right)+\tau\left(s+\nu_{0}\right)}{\sigma\left(s+\nu_{0}+1\right)} .
$$

Thus, for all $\nu_{i} \geq \nu_{0}$

$$
\rho_{\nu_{i}}(s)=\left[\sigma\left(s+\nu_{0}\right)+\tau\left(s+\nu_{0}\right)\right] \cdots\left[\sigma\left(s+\nu_{i}-1\right)+\tau\left(s+\nu_{i}-1\right)\right] \rho_{\nu_{0}}(s) .
$$

Using the last formula we obtain

$$
\sum_{i=1}^{3} A_{i}(z) \Phi_{\nu_{i} \mu_{i}}(z)=\sum_{s=a}^{b-1} \frac{\rho_{\nu_{0}}(s)}{(s-z)_{\mu_{0}+1}} \Pi(s),
$$

\footnotetext{
${ }^{2}$ In some cases this condition is equivalent to the condition $\left.s^{k} \sigma(s) \rho_{\nu_{0}}(s)\right|_{s=a} ^{s=b}=0$, $k=0,1,2, \ldots$
} 
where

$$
\Pi(s)=\sum_{i=1}^{3} A_{i}(z)\left(s-z+\mu_{i}+1\right)_{\mu_{0}-\mu_{i}} \pi\left(s+\nu_{0}\right) \cdots \pi\left(s+\nu_{i}-1\right),
$$

is a polynomial in $s$ and $\pi(s)=\sigma(s)+\tau(s)$. To conclude the proof we will show that the polynomials $A_{i}, i=1,2,3$ can be chosen such that

$$
\frac{\rho_{\nu_{0}}(s)}{(s-z)_{\mu_{0}+1}} \Pi(s)=\Delta\left[\frac{\rho_{\nu_{0}+1}(s-1)}{(s-z)_{\mu_{0}}} Q(s)\right]=\Delta\left[\frac{\sigma(s) \rho_{\nu_{0}}(s)}{(s-z)_{\mu_{0}}} Q(s)\right],
$$

where $Q(s)$ is a polynomial in $s$. Rewriting (3.8) with the help of the above formula and using the boundary condition (3.5) we find the expression ${ }^{3}$

$$
\sum_{i=1}^{3} A_{i}(z) \Phi_{\nu_{i} \mu_{i}}(z)=0
$$

Let us show that this polynomial $Q$ and the polynomials $A_{i}$ always exists. In fact, a straightforward computations give

$$
\begin{aligned}
\Delta\left[\frac{\sigma(s) \rho_{\nu_{0}}(s)}{(s-z)_{\mu_{0}}} Q(s)\right]=\frac{\rho_{\nu_{0}}(s)}{(s-z)_{\mu_{0}+1}} \times \\
\quad\left[\tau_{\nu_{0}}(s)(s-z) Q(s)+\left[\tau_{\nu_{0}}(s)+\sigma(s)\right](s-z) \Delta Q(s)-\mu_{0} \sigma(s) Q(s)\right],
\end{aligned}
$$

from which the following expression connecting the polynomials $\Pi$ and $Q$ follows

$$
\Pi(s)=\left[\tau_{\nu_{0}}(s)(s-z)-\mu_{0} \sigma(s)\right] Q(s)+(s-z)\left[\tau_{\nu_{0}}(s)+\sigma(s)\right] \Delta Q(s) .
$$

From the above relation follows that the degree of $Q(s)$ is two less than the degree of $\Pi(s)$ : it follows from the fact that the degree of $\tau_{\nu_{0}}$ is less than or equal to 1 . In fact, equating the coefficients of powers on the two sides of the above equation, we find a system of linear equations in the coefficients of $Q(s)$ and the coefficients $A_{i}$ which has at least one unknown more than the number of equations. Notice that the coefficients of the unknowns are polynomials in $z$, so that after one coefficient is selected the remaining coefficients are rational functions of $z$, therefore after multiplying by the common denominator of the $A_{i}(z)$ we obtain the linear relation with polynomial coefficients. This completes the proof.

Remark 3.2. Notice that for the discrete polynomials (2.9) the condition (3.6) is automatically fulfilled, since the contour $C$ is closed and $\nu$ is a nonnegative integer, so Lemma 3.1 holds for any family of discrete polynomials of hypergeometric type. Notice also that Lemma (3.1) assures the existence of the non vanishing polynomials in (3.4) but does not give any method for

\footnotetext{
${ }^{3}$ In the case of functions $\Phi_{\nu \mu}$ of the form (3.2) the proof is the same. In fact, changing the sums over $s$ by the integral over $C$ and using the boundary condition (3.6) we obtain the result.
} 
finding them. Nevertheless, using (3.9) and (3.11), a constructive approach for finding the coefficients $A_{i}, i=1,2,3$ can explicitly be written down. This will be shown in a simple example connecting the $\Phi_{\nu \nu-1}, \Phi_{\nu \nu}$, and $\Phi_{\nu \nu+1}$, functions. In fact, for the case of classical polynomials of Jacobi, Laguerre, Hermite and Bessel, the corresponding Lemma [23, page 14] has been extensively used for deriving several recurrences relations and for getting the corresponding coefficients in a closed form in terms of the polynomials $\sigma$ and $\tau$ (see e.g. $[17,24]$, and references therein). For the discrete case this study is under way.

Example. Let us find the relation among $\Phi_{\nu \nu-1}, \Phi_{\nu \nu}$, and $\Phi_{\nu \nu+1}$. In this case since $\operatorname{deg}(\Pi)=2, Q(z)=q_{0}$ is constant, and therefore (3.11) becomes $A_{1}(z)(s-z+\nu)_{2}+A_{2}(z)(s-z+\nu+1)+A_{3}=\left[\tau_{\nu_{0}}(s)(s-z)-\mu_{0} \sigma(s)\right] q_{0}$.

Expanding both sides in powers of $s-z$ and comparing coefficients we obtain

$$
A_{1}(z) \Phi_{\nu \nu-1}(z)+A_{2}(z) \Phi_{\nu \nu}(z)+A_{3}(z) \Phi_{\nu \nu+1}(z)=0
$$

where

$$
\begin{aligned}
& A_{1}(z)=\tau^{\prime}+(\nu-1) \frac{\sigma^{\prime \prime}}{2}, \quad A_{2}(z)=\tau(z)-\sigma^{\prime}(z)+\nu\left(\tau^{\prime}+\nu \frac{\sigma^{\prime \prime}}{2}\right), \\
& A_{3}(z)=-(\nu+1)\left[\sigma(z)+\nu\left(\tau^{\prime}+(\nu-1) \frac{\sigma^{\prime \prime}}{2}\right)\right] .
\end{aligned}
$$

To conclude this section we write down the following two straightforward identities for the functions $\Phi_{\nu \mu}$

$$
\Delta \Phi_{\nu \mu}(s)=(\mu+1) \Phi_{\nu \mu+1}(s+1),
$$

and

$$
\nabla \Phi_{\nu \mu}(s)=(\mu+1) \Phi_{\nu \mu+1}(s) .
$$

Recurrences involving the solutions $y_{\nu}$. Let us now establish the following relevant relation

$$
\Delta^{k} y_{\nu}(s)=\frac{C_{\nu}^{(k)}}{\rho_{k}(s)} \Phi_{\nu \nu-k}(s), \quad C_{\nu}^{(k)}=C_{\nu} \prod_{m=0}^{k-1}\left[\tau^{\prime}+(\nu+m-1) \frac{\sigma^{\prime \prime}}{2}\right] .
$$

This relation is valid for solutions of the form (2.3) and (2.5) of the difference equation (2.1). For the sake of simplicity we present here only the proof for the case of discrete polynomials. In this case (3.3) becomes

$$
P_{n}(s)=\frac{C_{n}}{\rho(s)} \Phi_{n n}(s) .
$$

Comparing the last formula with the integral representation (2.9) we deduce that $C_{n}=B_{n} n ! /(2 \pi i)$.

Next, using the Cauchy integral

$$
f(s)=\frac{1}{2 \pi i} \int_{C} \frac{f(z)}{z-s} d z,
$$


it follows that

$$
\nabla^{n-k} f(s)=\frac{(n-k) !}{2 \pi i} \int_{C} \frac{f(z)}{(z-s)_{n-k+1}} d z .
$$

Then, using the Rodrigues formula (2.11) for the $k$-th differences $\Delta^{k} P_{n}(s)$ we obtain the integral representation

$$
\Delta^{k} P_{n}(s)=\frac{A_{n k} B_{n}(n-k) !}{\rho_{k}(s) 2 \pi i} \int_{C} \frac{\rho_{n}(z)}{(z-s)_{n-k+1}} d z=\frac{C_{n}(n-k) ! A_{n k}}{n ! \rho_{k}(s)} \Phi_{n n-k}(s),
$$

from which, using (2.12), the relation (3.15) follows.

In the following $y_{n}^{(k)}(s)$ denotes the $k$-th differences $\Delta^{k} y_{n}(s)$.

Theorem 3.3. Under the same conditions as in Lemma 3.1, any three functions $y_{n_{i}}^{\left(k_{i}\right)}(s), i=1,2,3$ are connected by a relation of the form

$$
\sum_{i=1}^{3} B_{i}(s) y_{n_{i}}^{\left(k_{i}\right)}(s)=0
$$

with polynomials coefficients $B_{i}(s), i=1,2,3$.

Proof. From Lemma 3.1 we know that there exists three polynomials $A_{i}(s)$, $i=1,2,3$ such that

$$
\sum_{i=1}^{3} A_{i}(s) \Phi_{n_{i}, n_{i}-k_{i}}(s)=0 .
$$

Then, using the relation (3.15) we find

$$
\sum_{i=1}^{3} A_{i}(s)\left(C_{\nu}^{(k)}\right)^{-1} \rho_{k_{i}}(s) y_{n_{i}}^{\left(k_{i}\right)}(s)=0 .
$$

Now, dividing the last expression by $\rho_{k_{0}}(s), k_{0}=\min \left\{k_{1}, k_{2}, k_{3}\right\}$, and using (3.7) $\rho_{k_{i}}(s) / \rho_{k_{0}}(s)=\pi\left(s+k_{0}\right) \cdots \pi\left(s+k_{i}-1\right)$, we obtain

$$
\sum_{i=1}^{3} B_{i}(s) y_{n_{i}}^{\left(k_{i}\right)}(s)=0, \quad B_{i}(s)=A_{i}(s)\left(C_{\nu}^{(k)}\right)^{-1} \pi\left(s+k_{0}\right) \cdots \pi\left(s+k_{i}-1\right),
$$

which completes the proof.

Corollary 3.4. Under the same conditions as in Lemma 3.1, the following $\Delta$-ladder-type relations hold

$$
B_{1}(s) y_{n}(s)+B_{2}(s) \Delta y_{n}(s)+B_{3}(s) y_{n+m}(s)=0, \quad m \in \mathbb{Z},
$$

with polynomials coefficients $B_{i}(s), i=1,2,3$.

Proof. It is sufficient to put $k_{1}=k_{3}=0, k_{2}=1, n_{1}=n_{2}=n$ and $n_{3}=n+m$ in (3.16). 
Notice that for the case $m= \pm 1$ (3.17) becomes

$$
\begin{aligned}
& B_{1}(s) y_{n}(s)+B_{2}(s) \Delta y_{n}(s)+B_{3}(s) y_{n+1}(s)=0, \\
& \widetilde{B}_{1}(s) y_{n}(s)+\widetilde{B}_{2}(s) \Delta y_{n}(s)+\widetilde{B}_{3}(s) y_{n-1}(s)=0,
\end{aligned}
$$

with polynomials coefficients $B_{i}(s)$ and $\widetilde{B}_{i}(s), i=1,2,3$. The above relations (3.18) and (3.19) are usually called raising and lowering operators, respectively, for the functions $y_{n}$.

Let us now obtain raising and lowering operators for the functions $y_{\nu}$ but associated to the $\nabla$ operators.

We start by applying the operator $\nabla$ to the expression (3.3)

$$
\nabla y_{\nu}(s)=C_{\nu} \Phi_{\nu \nu}(s)\left(\frac{1}{\rho(s)}-\frac{1}{\rho(s-1)}\right)+\frac{C_{\nu}}{\rho(s-1)} \nabla \Phi_{\nu \nu}(s),
$$

or, equivalently,

$$
\nabla \Phi_{\nu \nu}(s)=\frac{\rho(s-1)}{C_{\nu}} \nabla y_{\nu}(s)-\Phi_{\nu \nu}(s)\left(\frac{\rho(s-1)}{\rho(s)}-1\right) .
$$

Next we use (3.4) with $\nu_{1}=\mu_{1}=\nu_{2}=\nu, \mu_{2}=\nu+1$, and $\nu_{3}=\mu_{3}=\nu+m$ and substitute the above formula as well as (3.14) to obtain the relation

$$
\begin{aligned}
A_{1}(s) \Phi_{\nu \nu}(s)+\frac{A_{2}(s)}{\nu+1} & {\left[\frac{\rho(s-1)}{C_{\nu}} \nabla y_{\nu}(s)-\left(\frac{\rho(s-1)}{\rho(s)}-1\right) \Phi_{\nu \nu}(s)\right] } \\
& +A_{3}(s) \Phi_{\nu+m, \nu+m}(s)=0 .
\end{aligned}
$$

Finally, using the Pearson-type equation $(2.7), \rho(s-1) / \rho(s)=\sigma(s) /[\sigma(s-$ $1)+\tau(s-1)]$, and multiplying the last expression by $C_{\nu}[\tau(s-1)+\sigma(s-$ $1)] / \rho(s)$ yields

$$
C_{1}(s) y_{\nu}(s)+C_{2}(s) \nabla y_{\nu}(s)+C_{3}(s) y_{\nu+m}(s)=0,
$$

where

$$
\begin{aligned}
& C_{1}(s)=(\nu+1)[\tau(s-1)+\sigma(s-1)] A_{1}(s)+[\sigma(s)-\tau(s-1)-\sigma(s-1)] A_{2}(s), \\
& C_{2}(s)=\sigma(s) A_{2}(s), \quad C_{3}(s)=(\nu+1)[\sigma(s-1)+\tau(s-1)] C_{\nu} C_{\nu+m}^{-1} A_{3}(s),
\end{aligned}
$$

i.e., we have proven the following

Theorem 3.5. Under the same conditions as in Lemma 3.1, the functions $y_{\nu}(s)$ satisfy the $\nabla$-ladder-type relations (3.20).

If we now choose $m= \pm 1$ we find the raising and lowering operators, respectively, for the functions $y_{n}$ associated with the $\nabla$-operator

$$
\begin{aligned}
& C_{1}(s) y_{n}(s)+C_{2}(s) \nabla y_{n}(s)+C_{3}(s) y_{n+1}(s)=0, \\
& \widetilde{C}_{1}(s) y_{n}(s)+\widetilde{C}_{2}(s) \nabla y_{n}(s)+\widetilde{C}_{3}(s) y_{n-1}(s)=0,
\end{aligned}
$$

with polynomials coefficients $C_{i}(s)$ and $\widetilde{C}_{i}(s), i=1,2,3$. 
Remark 3.6. Obviously the formulas (2.14)-(2.18) from section 2.2 are particular cases of the general expressions for the functions $y_{\nu}(s)$.

\section{Applichtions to Discrete systems}

As we already mentioned in the introduction, several important discrete systems (e.g. discrete oscillators) can be described using the solutions of the difference equation of hypergeometric type (2.1). In fact, in several of these models the corresponding wave functions have the form

$$
\psi(z):=\frac{\sqrt{\rho(z)}}{d_{n}} P_{n}(z),
$$

where $P_{n}$ is a classical discrete orthogonal polynomial of Hahn, Meixner, Kravchuk and Charlier, $d_{n}$ is the norm of $P_{n}$ and $\rho$ the corresponding weight function.

4.1. Three-term recurrence relations. Since $P_{n}$ can be expressed by (2.9), we can use theorem 3.3 that assures the existence of the three-term recurrence relation

$$
A_{1} P_{n+1}(z)+A_{2} P_{n}(z)+A_{3} P_{n-1}(z)=0 .
$$

But comparing it with (2.13) yields $A_{1}(z)=1, A_{2}(z)=\beta_{n}-z$ and $A_{3}(z)=$ $\gamma_{n}$, therefore, we may write

$$
\frac{d_{n+1}}{d_{n}} \psi_{n+1}(z)+\left(\beta_{n}-z\right) \psi_{n}(z)+\gamma_{n} \frac{d_{n-1}}{d_{n}} \psi_{n-1}(z) .
$$

Charlier case. The Charlier functions are defined by

$$
\psi_{n}^{\mu}(z)=\sqrt{\frac{e^{-\mu} \mu^{z-n}}{\Gamma(z+1) n !}} C_{n}^{\mu}(z), \quad n \geq 0 .
$$

Using the main data for the Charlier polynomials (see Table 3) we obtain

$$
\sqrt{(n+1) \mu} \psi_{n+1}^{\mu}(z)+[(n+\mu)-z] \psi_{n}^{\mu}(z)+\sqrt{n \mu} \psi_{n-1}^{\mu}(z) .
$$

Meixner case. In the Meixner case we have

$$
\psi_{n}^{\gamma, \mu}(z)=\mu^{(z-n) / 2}(1-\mu)^{\gamma / 2+n} \sqrt{\frac{\Gamma(\gamma+z)}{\Gamma(\gamma) \Gamma(z+1) n !(\gamma)_{n}}} M_{n}^{\gamma, \mu}(z), \quad n \geq 0,
$$

thus

$$
\begin{gathered}
\sqrt{(n+1) \mu(\gamma+n)} \psi_{n+1}^{\gamma, \mu}(z)+[n(1+\mu)+\mu \gamma-(1-\mu) z] \psi_{n}^{\gamma, \mu}(z) \\
+\sqrt{n \mu(\gamma+n-1)} \psi_{n-1}^{\gamma, \mu}(z) .
\end{gathered}
$$


Kravchuk case. The Kravchuk functions defined by

$$
\psi_{n}^{p}(z)=p^{(z-n) / 2}(1-p)^{(N-n-z) / 2} \sqrt{\frac{(N-n) !}{n ! \Gamma(z+1) \Gamma(N-z+1)}} K_{n}^{p}(z, N),
$$

are a finite set of orthogonal functions $n=0,1, \ldots N$. For these functions (4.2) becomes

$$
\begin{aligned}
\sqrt{(n+1) p(1-p)(N-n)} & \psi_{n+1}^{p}(z)+[N p+(1-2 p) n-z] \psi_{n}^{p}(z) \\
& +\sqrt{n p(1-p)(N-n+1)} \psi_{n-1}^{p}(z)=0 .
\end{aligned}
$$

Hahn case. Finally, for the Hahn functions

$$
\psi_{n}^{\alpha, \beta}(z)=\sqrt{\frac{\Gamma(N+\alpha-z) \Gamma(\beta+z+1)(\alpha+\beta+2 n+1) \Gamma(N-n) \Gamma(\alpha+\beta+n+1)(\alpha+\beta+n+1)_{n}^{2}}{\Gamma(N-z) \Gamma(z+1) n ! \Gamma(\alpha+n+1) \Gamma(\beta+n+1) \Gamma(\alpha+\beta+N+n+1)}} h_{n}^{\alpha, \beta}(z)
$$

we have

$$
a_{n} \psi_{n+1}^{\alpha, \beta}(z)+\left(b_{n}-z\right) \psi_{n}^{\alpha, \beta}(z)+a_{n-1} \psi_{n-1}^{\alpha, \beta}(z)=0
$$

where

$$
a_{n}=\sqrt{\frac{n(N-n)(\alpha+n)(\beta+n)(\alpha+\beta+n)(\alpha+\beta+N+n)}{(\alpha+\beta+2 n-1)(\alpha+\beta+2 n)^{2}(\alpha+\beta+2 n+1)}}
$$

and

$$
b_{n}=\frac{(\beta+1)(N-1)(\alpha+\beta)+n(2 N+\alpha-\beta-2)(\alpha+\beta+n+1)}{(\alpha+\beta+2 n)(\alpha+\beta+2 n+2)} .
$$

4.2. Ladder-type Relations. Let us look for relations involving the operators $\nabla$ and $\Delta$. Since $P_{n}(z)=d_{n} \psi_{n}(z) / \sqrt{\rho(z)}$, we have

$$
\nabla P_{n}(z)=\frac{d_{n}}{\sqrt{\rho(z-1)}} \nabla \psi_{n}(z)+d_{n} \psi_{n}(z)\left(\frac{1}{\sqrt{\rho(z)}}-\frac{1}{\sqrt{\rho(z-1)}}\right) .
$$

On the other hand, Theorem 3.5 guarantees that for any integer $m$ there exist polynomials $A_{1}, A_{2}$ and $A_{3}$ which are not all three vanishing such that

$$
A_{1} P_{n}(z)+A_{2} \nabla P_{n}(z)+A_{3} P_{n+m}(z)=0 .
$$

Substituting (4.11) in (4.12) we get

$$
\left[A_{1}+A_{2}\left(1-\sqrt{\frac{\rho(z)}{\rho(z-1)}}\right)\right] \psi_{n}(z)+A_{2} \sqrt{\frac{\rho(z)}{\rho(z-1)}} \nabla \psi_{n}(z)+A_{3} \frac{d_{n+m}}{d_{n}} \psi_{n+m}(z)=0 .
$$

In a similar way, application of $\Delta$ to $P_{n}(z)=d_{n} \psi_{n}(z) / \sqrt{\rho(z)}$ gives

$$
\Delta P_{n}(z)=\frac{d_{n}}{\sqrt{\rho(z+1)}} \Delta \psi_{n}(z)+d_{n}\left(\frac{1}{\sqrt{\rho(z+1)}}-\frac{1}{\sqrt{\rho(z)}}\right) \psi_{n}(z)
$$


and consequently, substituting (4.14) into (3.17) gives

$$
\begin{array}{r}
{\left[B_{1}+B_{2}\left(\sqrt{\frac{\rho(z)}{\rho(z+1)}}-1\right)\right] \psi_{n}(z)+B_{2} \sqrt{\frac{\rho(z)}{\rho(z+1)}} \Delta \psi_{n}(z)} \\
+B_{3} \frac{d_{n+m}}{d_{n}} \psi_{n+m}(z)=0
\end{array}
$$

Let us point out that for getting the polynomial coefficients $B_{i}, i=1,2,3$ in (3.17) (or $A_{i}, i=1,2,3$ in (4.12)) we will follow the idea in [16]. Given $m$, the idea is to identify the corresponding relation (3.17) (or (4.12)) with one of the known expressions (2.13)-(2.18) for the classical polynomials. In doing so, after $m$ is choosen and fixed, (3.17) (or (4.12)) may or may not transform into one of the known formulas (2.13)-(2.18). In the first situation, which is the simplest one, we can identify directly the coefficients $B_{i}, i=1,2,3$ (or $A_{i}, i=1,2,3$ ) comparing the formula (3.17) (or (4.12)) with one of the known expressions (2.13)-(2.18). In the second one, in general we need to combine (3.17) (or (4.12)) with a certain combination of two or more formulas (2.13)-(2.18) to obtain the unknown polynomials $B_{i}, i=1,2,3$ (or $A_{i}, i=1,2,3$ ). In the following examples we show how this works in the first situation. The more "complex" cases will be considered in the next subsection 4.3.2.

Charlier case. Substituting (4.3) into (4.13) and (4.15), gives, respectively

$$
\begin{aligned}
{\left[\sqrt{z} A_{1}+(\sqrt{z}-\sqrt{\mu}) A_{2}\right] \psi_{n}^{\mu}(z) } & +A_{2} \sqrt{\mu \nabla \psi_{n}^{\mu}(z)} \\
& +A_{3} \sqrt{z \mu^{m}(n+1)_{m}} \psi_{n+m}^{\mu}(z)=0 \\
{\left[\sqrt{\mu} B_{1}+(\sqrt{z+1}-\sqrt{\mu}) B_{2}\right] } & \psi_{n}^{\mu}(z)+B_{2} \sqrt{z+1} \Delta \psi_{n}^{\mu}(z) \\
+ & B_{3} \sqrt{\mu^{m+1}(n+1)_{m}} \psi_{n+m}^{\mu}(z)=0
\end{aligned}
$$

Let us remind here that $(a)_{k}$ denotes the Pochhammer symbol (2.8).

Now we proceed by choosing particular values for the parameter $m \in \mathbb{Z}$.

- We start with the case $m=-1$. Then (4.12) becomes

$$
A_{1} C_{n}^{\mu}(z)+A_{2} \nabla C_{n}^{\mu}(z)+A_{3} C_{n-1}^{\mu}(z)=0 .
$$

Comparing the above equation with (2.16) we find $A_{1}=-n, A_{2}=z$, $A_{3}=-n \mu$, and therefore (4.16) becomes

$$
(z-\sqrt{\mu z}-n) \psi_{n}^{\mu}(z)+\sqrt{\mu z} \nabla \psi_{n}^{\mu}(z)-\sqrt{n \mu} \psi_{n-1}^{\mu}(z)=0 .
$$

On the other hand, (3.17) becomes

$$
B_{1} C_{n}^{\mu}(z)+B_{2} \Delta C_{n}^{\mu}(z)+B_{3} C_{n-1}^{\mu}(z)=0
$$

so, comparing with (2.17) gives $B_{1}=0, B_{2}=1, B_{3}=-n$. Therefore, (4.17) gives

$$
(\sqrt{z+1}-\sqrt{\mu}) \psi_{n}^{\mu}(z)+\sqrt{z+1} \Delta \psi_{n}^{\mu}(z)-\sqrt{n} \psi_{n-1}^{\mu}(z)=0 .
$$


- Analogously, for the case $m=1,(4.12)$ becomes

$$
A_{1} C_{n}^{\mu}(z)+A_{2} \nabla C_{n}^{\mu}(z)+A_{3} C_{n+1}^{\mu}(z)=0 .
$$

Now, comparing with (2.14) gives $A_{1}=\mu-z, A_{2}=z, A_{3}=1$, thus, (4.16) becomes

$$
(\sqrt{\mu}-\sqrt{z}) \psi_{n}^{\mu}(z)+\sqrt{z} \nabla \psi_{n}^{\mu}(z)+\sqrt{n+1} \psi_{n+1}^{\mu}(z)=0,
$$

In this case (3.17) has the form

$$
B_{1} C_{n}^{\mu}(z)+B_{2} \Delta C_{n}^{\mu}(z)+B_{3} C_{n+1}^{\mu}(z)=0 .
$$

If we compare the above expression with (2.15) we find $B_{1}=\mu+n-z$, $B_{2}=\mu, B_{3}=1$, so (4.17) transforms to

$$
\begin{aligned}
{[(\mu-z)(\mu+n-z)-\mu(\sqrt{\mu}-\sqrt{z+1})] } & \psi_{n}^{\mu}(z)+\mu \sqrt{z+1} \Delta \psi_{n}^{\mu}(z) \\
& +\mu \sqrt{n+1} \psi_{n+1}^{\mu}(z)=0 .
\end{aligned}
$$

Meixner case. Substituting (4.5) into (4.13) and (4.15), we get, respectively

$$
\begin{aligned}
{\left[\sqrt{z} A_{1}+\right.} & \left.A_{2}(\sqrt{z}-\sqrt{\mu(\gamma-1+z)})\right] \psi_{n}^{\gamma, \mu}(z)+A_{2} \sqrt{\mu(\gamma-1+z)} \nabla \psi_{n}^{\gamma, \mu}(z) \\
& +A_{3} \sqrt{\frac{z \mu^{m}(n+1)_{m}(\gamma+n)_{m}}{(1-\mu)^{2 m}}} \psi_{n+m}^{\gamma, \mu}(z)=0
\end{aligned}
$$

and

$$
\begin{gathered}
{\left[\sqrt{\mu(\gamma+z)} B_{1}+B_{2}(\sqrt{z+1}-\sqrt{\mu(\gamma+z)})\right] \psi_{n}^{\gamma, \mu}(z)+B_{2} \sqrt{z+1} \Delta \psi_{n}^{\gamma, \mu}(z)} \\
+B_{3} \sqrt{\frac{(\gamma+z) \mu^{m+1}(n+1)_{m}(\gamma+n)_{m}}{(1-\mu)^{2 m}}} \psi_{n+m}^{\gamma, \mu}(z)=0 .
\end{gathered}
$$

Now we proceed by choosing particular values for the parameter $m \in \mathbb{Z}$.

- For $m=-1$ (4.12) becomes

$$
A_{1} M_{n}^{\gamma, \mu}(z)+A_{2} \nabla M_{n}^{\gamma, \mu}(z)+A_{3} M_{n-1}^{\gamma, \mu}(z)=0 .
$$

Comparing with (2.16) we get $A_{1}=n, A_{2}=-z, A_{3}=\frac{n \mu(n-1+\gamma)}{1-\gamma}$, so (4.22) becomes

$$
\begin{aligned}
& {[n \sqrt{z}-z(\sqrt{z}-\sqrt{\mu(\gamma-1+z)})] \psi_{n}^{\gamma, \mu}(z)} \\
& -z \sqrt{\mu(\gamma-1+z)} \nabla \psi_{n}^{\gamma, \mu}(z)+\sqrt{z n \mu(n-1+\gamma)} \psi_{n-1}^{\gamma, \mu}(z)=0
\end{aligned}
$$

In this case (3.17) has the form

$$
B_{1} M_{n}^{\gamma, \mu}(z)+B_{2} \Delta M_{n}^{\gamma, \mu}(z)+B_{3} M_{n-1}^{\gamma, \mu}(z)=0 .
$$


Comparing the last equation with (2.17) yields $B_{1}=n, B_{2}=-z-\gamma$, and $B_{3}=\frac{n(n-1+\gamma)}{1-\mu}$, therefore (4.23) gives

$$
\begin{aligned}
& {[n \sqrt{\mu}-\sqrt{z+\gamma}(\sqrt{z+1}-\sqrt{\mu(\gamma+z)})] \psi_{n}^{\gamma, \mu}(z)} \\
& -\sqrt{(z+1)(z+\gamma)} \Delta \psi_{n}^{\gamma, \mu}(z)+\sqrt{n(n-1+\gamma)} \psi_{n-1}^{\gamma, \mu}(z)=0 .
\end{aligned}
$$

- Choosing $m=1,(4.12)$ becomes

$$
A_{1} M_{n}^{\gamma, \mu}(z)+A_{2} \nabla M_{n}^{\gamma, \mu}(z)+A_{3} M_{n+1}^{\gamma, \mu}(z)=0 .
$$

A solution now is (compare with (2.14))

$$
A_{1}=\mu(\gamma+n)-(1-\mu) z, \quad A_{2}=z, \quad A_{3}=1-\mu,
$$

thus (4.22) becomes

$$
\begin{aligned}
& {[(\mu(\gamma+n+z)-\sqrt{\mu z(\gamma-1+z)})] \psi_{n}^{\gamma, \mu}(z)} \\
& \quad+\sqrt{\mu z(\gamma-1+z)} \nabla \psi_{n}^{\gamma, \mu}(z)+\sqrt{\mu(n+1)(\gamma+n)} \psi_{n+1}^{\gamma, \mu}(z)=0 .
\end{aligned}
$$

In this case (3.17) takes the form

$$
B_{1} M_{n}^{\gamma, \mu}(z)+B_{2} \Delta M_{n}^{\gamma, \mu}(z)+B_{3} M_{n+1}^{\gamma, \mu}(z)=0,
$$

which comparing with (2.15) gives $B_{1}=\mu(z+\gamma)+n-z, B_{2}=\mu(z+\gamma)$, and $B_{3}=1-\mu$. Thus (4.23) gives

$$
\begin{aligned}
& {\left[\sqrt{\mu^{-1}}(\mu(\gamma+z)+n-z)+\sqrt{z+\gamma}(\sqrt{z+1}-\sqrt{\mu(\gamma+z)})\right] \psi_{n}^{\gamma, \mu}(z)} \\
& +\sqrt{(z+1)(z+\gamma)} \Delta \psi_{n}^{\gamma, \mu}(z)+\sqrt{(n+1)(\gamma+n)} \psi_{n+1}^{\gamma, \mu}(z)=0
\end{aligned}
$$

Kravchuk case. In this case using (4.7), (4.13) and (4.15) we have

$$
\begin{gathered}
{\left[\sqrt{(1-p) z} A_{1}+A_{2}(\sqrt{(1-p) z}-\sqrt{p(N+1-z)})\right] \psi_{n}^{p}(z)} \\
+A_{2} \sqrt{p(N+1-z)} \nabla \psi_{n}^{p}(z) \\
+A_{3} \sqrt{z(n+1)_{m}(N-n-m+1)_{m} p^{m}(1-p)^{m+1}} \psi_{n+m}^{p}(z)=0, \\
{\left[\sqrt{p(N-z)} A_{1}+A_{2}(\sqrt{(1-p)(z+1)}-\sqrt{p(N-z)})\right] \psi_{n}^{p}(z)} \\
+A_{3} \sqrt{(1-p)(z+1)} \Delta \psi_{n}^{p}(z) \\
+A_{2} \sqrt{(N-z)(n+1)_{m}(N-n-m+1)_{m} p^{m+1}(1-p)^{m}} \psi_{n+m}^{p}(z)=0 .
\end{gathered}
$$

- For $m=-1,(4.12)$ becomes

$$
A_{1} K_{n}^{p}(z)+A_{2} \nabla K_{n}^{p}(z)+A_{3} K_{n-1}^{p}(z)=0,
$$

for which the solution is (see (2.16))

$$
A_{1}=n, \quad A_{2}=-z, \quad A_{3}=p n(N-n+1),
$$


and therefore (4.28) becomes

$$
\begin{aligned}
& {[(\sqrt{(1-p) z}-\sqrt{p(N+1-z)}) z-n \sqrt{(1-p) z}] \psi_{n}^{p}(z)} \\
& +\sqrt{p(N+1-z)} z \nabla \psi_{n}^{p}(z)-\sqrt{z p n(N-n+1)} \psi_{n-1}^{p}(z)=0 .
\end{aligned}
$$

Now (3.17) gives

$$
B_{1} K_{n}^{p}(z) B_{2} \Delta K_{n}^{p}(z)+B_{3} K_{n-1}^{p}(z)=0,
$$

from which, comparing with (2.17), we find $B_{1}=n, B_{2}=N-z$, and $B_{3}=-n(N-n+1)(1-p)$. Then (4.29) yields

$$
\begin{aligned}
& {\left[n \sqrt{p}+\sqrt{N-z}(\sqrt{(1-p)(z+1)}-\sqrt{p(N-z)}] \psi_{n}^{p}(z)\right.} \\
& +\sqrt{(1-p)(z+1)(N-z)} \Delta \psi_{n}^{p}(z)-\sqrt{n(N-n+1)(1-p)} \psi_{n-1}^{p}(z)=0 .
\end{aligned}
$$

- Now, for $m=1,(4.12)$ has the form

$$
A_{1} K_{n}^{p}(z)+A_{2} \nabla K_{n}^{p}(z)+A_{3} K_{n+1}^{p}(z)=0
$$

for which $A_{1}=(N-n) p-z, A_{2}=(1-p) z, A_{3}=1$, (see (2.14)) so (4.28) becomes

$$
\begin{aligned}
& {\left[\sqrt{z} \frac{(N-n) p-z}{\sqrt{p(1-p)}}+\left(\sqrt{\frac{1-p}{p}} z-\sqrt{N+1-z}\right) z\right] \psi_{n}^{p}(z)} \\
& \quad+z \sqrt{N+1-z} \nabla \psi_{n}^{p}(z)+\sqrt{(n+1)(N-n) z} \psi_{n+1}^{p}(z)=0 .
\end{aligned}
$$

Since for this case (3.17) has the form

$$
B_{1} K_{n}^{p}(z)+B_{2} \Delta K_{n}^{p}(z)+B_{3} K_{n+1}^{p}(z)=0
$$

then, comparing with (2.15), $B_{1}=(N-n) p+n-z, B_{2}=p(N-z)$, and $B_{3}=1$, so (4.29) transforms into

$$
\begin{aligned}
& \left\{\frac{\sqrt{p(N-z)}}{1-p}[(N-n) p+n-z]+[\sqrt{(1-p)(z+1)}-\sqrt{p(N-z)}] z\right\} \psi_{n}^{p}(z) \\
& +z \sqrt{(1-p)(z+1)} \Delta \psi_{n}^{p}(z)+p \sqrt{(1-p)(n+1)(N-n)(N-z)} \psi_{n+1}^{p}(z)=0 .
\end{aligned}
$$

Hahn case. As in the previous cases we substitute (4.9) in (4.13) and (4.15) to obtain, respectively, the expressions

$$
\begin{aligned}
{\left[A_{1} \sqrt{\sigma(z)}\right.} & \left.+A_{2} g(z)\right] \psi_{n}^{\alpha, \beta}(z)+A_{2} \sqrt{\pi(z-1)} \nabla \psi_{n}^{\alpha, \beta}(z) \\
& +A_{3} \sqrt{\sigma(z)} \frac{d_{n+m}}{d_{n}} \psi_{n+m}^{\alpha, \beta}(z)=0,
\end{aligned}
$$


and

$$
\begin{aligned}
{\left[B_{1} \sqrt{\pi(z)}+\right.} & \left.B_{2} g(z+1)\right] \psi_{n}^{\alpha, \beta}(z)+B_{2} \sqrt{\sigma(z+1)} \Delta \psi_{n}^{\alpha, \beta}(z) \\
& +B_{3} \sqrt{\pi(z)} \frac{d_{n+m}}{d_{n}} \psi_{n+m}^{\alpha, \beta}(z)=0
\end{aligned}
$$

where $\sigma(z)=z(N+\alpha-z), \pi(z)=(z+\beta+1)(N-z-1)$, and $g(z)=$ $\sqrt{\sigma(z)}-\sqrt{\pi(z-1)}$. In the following we will use also the following notation

$$
f(z)=\sqrt{\sigma(z) \pi(z-1)}=\sqrt{z(N+\alpha-z)(z+\beta)(N-z)},
$$

and

$$
\theta_{n}=\sqrt{\frac{n(N-n)(\alpha+n)(\beta+n)(\alpha+\beta+n)(\alpha+\beta+N+n)}{(\alpha+\beta+2 n-1)(\alpha+\beta+2 n)^{2}(\alpha+\beta+2 n+1)}} .
$$

Using the same technique as in the previous cases we arrive at the following expressions

- $m=-1$

$\left[n\left(\epsilon_{n}-z\right)-\sqrt{\sigma(z)} g(z)\right] \psi_{n}^{\alpha, \beta}(z)-f(z) \nabla \psi_{n}^{\alpha, \beta}(z)+(\alpha+\beta+2 n+1) \theta_{n} \psi_{n-1}^{\alpha, \beta}(z)=0$,

where $\epsilon_{n}=\frac{(\beta+1)(N-1)(\alpha+\beta)+(\alpha+\beta+n+1)[(\alpha+\beta)(\alpha+1+2 n)+(n+N)(\alpha-\beta+2 n)]}{(\alpha+\beta+2 n)(\alpha+\beta+2 n+2)}$ and

$$
\begin{array}{r}
{\left[n\left(z+\varsigma_{n}\right)+\sqrt{\pi(z)} g(z+1)\right] \psi_{n}^{\alpha, \beta}(z)+f(z+1) \Delta \psi_{n}^{\alpha, \beta}(z)} \\
-(\alpha+\beta+2 n+1) \theta_{n} \psi_{n-1}^{\alpha, \beta}(z)=0
\end{array}
$$

where $\varsigma_{n}=\alpha+\beta+n+1-\frac{(\alpha+\beta)(\alpha+\beta+N+n)}{\alpha+\beta+2 n}$.

- $m=1$

$$
\begin{array}{r}
{\left[(\alpha+\beta+n+1)\left(\varepsilon_{n}-z\right)-\sqrt{\sigma(z)} g(z)\right] \psi_{n}^{\alpha, \beta}(z)-f(z) \nabla \psi_{n}^{\alpha, \beta}(z)} \\
+(\alpha+\beta+1) \theta_{n+1} \psi_{n+1}^{\alpha, \beta}(z)=0
\end{array}
$$

where

$$
\varepsilon_{n}=\frac{(\alpha+\beta)[n(\alpha+1+2 n)+(N-1)(\beta+1+2 n)]+n(\alpha-\beta)(\alpha+\beta+n+N+1)+2 n(n+1)(n+N-1)}{(\alpha+\beta+2 n)(\alpha+\beta+2 n+2)},
$$

and

$$
\begin{gathered}
{\left[(\alpha+\beta+1)\left(\xi_{n}-z\right)+\sqrt{\pi(z)} g(z+1)\right] \psi_{n}^{\alpha, \beta}(z)+f(z+1) \Delta \psi_{n}^{\alpha, \beta}(z)} \\
+(\alpha+\beta+2 n+1) \theta_{n+1} \psi_{n+1}^{\alpha, \beta}(z)=0
\end{gathered}
$$

where $\xi_{n}=n+\frac{(\beta+1)(N-1)+n(N-\beta-n-2)}{\alpha+\beta+2 n+2}$. 
4.3. Further examples. To conclude this work let us show how we can use the method presented here for finding some higher recurrence relations for the Charlier polynomials. Notice that in the following four examples one should use a combination of known relations of discrete orthogonal polynomials. In a similar way, we can proceed with the other families to obtain more recurrence relations.

4.3.1. Recurrences for Charlier polynomials. We start with some examples of the recurrences (3.16) connecting Charlier polynomials.

Choosing, in (3.16),

$$
n_{1}=n-1, n_{2}=n, n_{3}=n+1 ; \quad k_{1}=1, k_{2}=1, \quad k_{3}=0
$$

we find

$$
B_{1}(z) \Delta C_{n-1}^{\mu}(z)+B_{2}(z) \Delta C_{n}^{\mu}(z)+B_{3}(z) C_{n+1}^{\mu}(z)=0 .
$$

Hence, by (2.17), $\Delta C_{n}^{\mu}(z)=n C_{n-1}^{\mu}(z)$, it becomes into

$$
(n-1) B_{1}(z) C_{n-2}^{\mu}(z)+n B_{2}(z) C_{n-1}^{\mu}(z)+B_{3}(z) C_{n+1}^{\mu}(z)=0 .
$$

Using, now, the TTRR for the Charlier polynomials (cf. (2.13)), the last relation transforms into

$(n-1) B_{1}(z) C_{n-2}^{\mu}(z)+n\left[B_{2}(z)-\mu B_{3}(z)\right] C_{n-1}^{\mu}(z)+(z-n-\mu) B_{3}(z) C_{n}^{\mu}(z)=0$.

Therefore, comparing with (2.13), we obtain

$B_{1}(z)=n \mu(z-n-\mu), \quad B_{2}(z)=n \mu-(z-n-\mu)(z-n+1-\mu), \quad B_{3}(z)=n$,

which leads to

$n C_{n+1}^{\mu}(z)=[(z-n-\mu)(z-n+1-\mu)-n \mu] \Delta C_{n}^{\mu}(z)-n \mu(z-n-\mu) \Delta C_{n-1}^{\mu}(z)$.

Let us now choose,

$$
n_{1}=n-1, n_{2}=n, n_{3}=n+3 ; \quad k_{1}=0, \quad k_{2}=0, \quad k_{3}=2 .
$$

Then (3.16) reads

$$
B_{1}(z) C_{n-1}^{\mu}(z)+B_{2}(z) C_{n}^{\mu}(z)+B_{3}(z) \Delta^{2} C_{n+3}^{\mu}(z)=0 .
$$

Using now (2.17), it becomes into

$$
B_{1}(z) C_{n-1}^{\mu}(z)+B_{2}(z) C_{n}^{\mu}(z)+(n+3)(n+2) B_{3}(z) C_{n+1}^{\mu}(z)=0 .
$$

If we compare the last expression with the TTRR for Charlier polynomials (cf. (2.13)) one finds

$B_{1}(z)=n(n+2)(n+3) \mu, \quad B_{2}(z)=(n+2)(n+3)(n+\mu-z), \quad B_{3}(z)=1$.

Then,

$$
\Delta^{2} C_{n+3}^{\mu}(z)=(n+2)(n+3)(z-n-\mu) C_{n}^{\mu}(z)-n(n+2)(n+3) \mu C_{n-1}^{\mu}(z) .
$$


4.3.2. Higher order ladder-type relations for Charlier functions $\psi_{n}^{\mu}$. Let us now chose $m=-2$ in (4.16). Then (4.12) becomes

$$
A_{1} C_{n}^{\mu}(z)+A_{2} \nabla C_{n}^{\mu}(z)+A_{3} C_{n-2}^{\mu}(z)=0,
$$

which does not correspond to any of the known relations for the Charlier polynomials. To obtain the coefficients $A_{1}, A_{2}$ and $A_{3}$, we can proceed as follows: Using the TTRR (cf. (2.13)) for the Charlier polynomials

$$
z C_{n-1}^{\mu}(z)=C_{n}^{\mu}(z)+(n-1+\mu) C_{n-1}^{\mu}(z)+(n-1) \mu C_{n-2}^{\mu}(z),
$$

(4.40) transforms into

$$
\left(A_{1}-\frac{A_{3}}{(n-1) \mu}\right) C_{n}^{\mu}(z)+A_{2} \nabla C_{n}^{\mu}(z)+A_{3} \frac{x-n+1-\mu}{\mu(n-1)} C_{n-1}^{\mu}(z)=0 .
$$

Now comparing with (2.16) we obtain

$$
A_{1}(z)=-\frac{n(z-n+1)}{z-n+1-\mu}, \quad A_{2}(z)=z, \quad A_{3}=-\frac{n(n-1) \mu^{2}}{z-n+1-\mu} .
$$

Now, multiplying by $z-n+1-\mu$ we finally obtain

$$
-n(z-n+1) C_{n}^{\mu}(z)+z(z-n+1-\mu) \nabla C_{n}^{\mu}(z)-n(n-1) \mu^{2} C_{n-2}^{\mu}(z)=0 .
$$

Thus, we find for the functions $\psi_{n}^{\mu}(z)$ the relation

$$
\begin{aligned}
0=[- & n(z-n+1) \sqrt{z}+(\sqrt{z}-\sqrt{\mu}) z(z-\mu-n+1)] \psi_{n}^{\mu}(z) \\
& +\sqrt{\mu} z(z-\mu-n+1) \nabla \psi_{n}^{\mu}(z)-\mu \sqrt{z n(n-1)} \psi_{n-2}^{\mu}(z) .
\end{aligned}
$$

Similarly, substituting $m=2$ in (3.17) we find

$$
B_{1}(z) C_{n}^{\mu}(z)+B_{2}(z) \Delta C_{n}^{\mu}(z)+B_{3}(z) C_{n+2}^{\mu}(z)=0 .
$$

Now, using twice the TTRR (cf. (2.13))

$$
C_{n+1}^{\mu}(z)=(z-n-\mu) C_{n}^{\mu}(z)-n C_{n-1}^{\mu}(z)
$$

we obtain

$$
\begin{aligned}
& B_{2}(z) \Delta C_{n}^{\mu}(z)=n \mu(z-n-1-\mu) B_{3}(z) C_{n-1}^{\mu}(z) \\
& -\left\{B_{1}(z)+[(z-n-1-\mu)(z-n-\mu)-(n+1) \mu] B_{3}(z)\right\} C_{n}^{\mu}(z)
\end{aligned}
$$

Comparing (4.42) with (2.17) one finds

$$
B_{1}(z)=(n+1) \mu-(z-n-1-\mu)(z-n-\mu), \quad B_{2}(z)=\mu(z-n-1-\mu)
$$

and $B_{3}(z)=1$. This leads to the following relation for Charlier functions $\psi_{n}^{\mu}$

$$
\begin{aligned}
& \{\sqrt{\mu}[(n+1) \mu-(z-n-1-\mu)(z-n-\mu)]+ \\
& (\sqrt{z+1}-\sqrt{\mu}) \mu(z-n-1-\mu)\} \psi_{n}^{\mu}(z) \\
& +\mu(z-n-1-\mu) \sqrt{z+1} \Delta \psi_{n}^{\mu}(z)-\sqrt{\mu^{3}(n+1)(n+2)} \psi_{n+2}^{\mu}(z)=0,
\end{aligned}
$$

that corresponds to the ladder-type relation (4.17) with $m=2$. 
Concluding remarks. In this paper we present a simple, unified and constructive approach for finding linear recurrence relations for the difference hypergeometric-type functions, i.e., solutions of the hypergeometric difference equation (2.1), and apply the general results to some discrete models (e.g. discrete oscillators). Furthermore, the method described here is valuable for new situations, such as higher order recurrence relations and ladder-type relations for the classical discrete orthogonal polynomials.

Other important instances of discrete systems are the so-called $q$-oscillators (see e.g. $[3,6,7,15,21]$ and reference therein) that are related with the $q$-polynomials. For these cases only a few recurrences are known [4]. A more detailed study of these $q$-models is under way.

Acknowledgements. The authors thank J.S. Dehesa, J.C. Petronilho and A. Zarzo for interesting discussions. We also thank the unknown referees for their comments and remarks that help us to improve the paper. This research has been supported by the Programa de Acciones Integradas HispanoLusas HP2002-065 \& E-6/03. The authors were also partially supported by the the DGES grant BFM 2003-06335-C03-01 and PAI grant FQM-0262 (RAN); the DGES grant BFM2001-3878-C02 and PAI grant FQM-0207 (NRQ); and CMUC (JLC).

\section{REFERENCES}

[1] R. Álvarez-Nodarse, Polinomios hipergemétricos y q-polinomios. Monografías del Seminario Matemático "García de Galdeano" Vol. 26. Prensas Universitarias de Zaragoza, Zaragoza, Spain, 2003.

[2] R. Álvarez-Nodarse, N. M. Atakishiyev, and R. S. Costas-Santos, Factorization of the hypergeometric-type difference equation on the uniform lattice. Electron. Trans. Numer. Anal. (to appear)

[3] R. Álvarez-Nodarse, N. M. Atakishiyev, and R. S. Costas-Santos, Factorization of the hypergeometric-type difference equation on the non-uniform lattices: dynamical algebra. J. Phys. A: Math. Gen. 38 (2005), 153-174.

[4] R. Álvarez-Nodarse, M. K. Atakishiyeva, and N. M. Atakishiyev, On a $q$-extension of the quantum harmonic oscillator. Preprint 2004.

[5] N. M. Atakishiyev, E.I. Jafarov, S.M. Nagiyev, and K.B. Wolf, Meixner oscillators. Revista Mexicana de Física 44(3) (1998), 235-244.

[6] N. M. Atakishiyev and S. K. Suslov, Difference analogs of the harmonic oscillator. Theor. Math. Phys. 85 (1991), 442-444.

[7] N. M. Atakishiyev and S. K. Suslov, A realization of the $q$-harmonic oscillator. Theor. Math. Phys. 87 (1991), 1055-1062.

[8] N. M. Atakishiyev, G. S. Pogosyan, and B. Wolf, Contraction of the finite onedimensional oscillator: I. The cartesian model. J. Phys. A: Math. Gen. 34 (2001), 9381-9398.

[9] N. M. Atakishiyev, G. S. Pogosyan, L. E. Vicent, and B. Wolf, Finite two-dimensional oscillator: II. The radial model. J. Phys. A: Math. Gen. 34 (2001), 9399-9415.

[10] N. M. Atakishiyev, G. S. Pogosyan, and B. Wolf, Contraction of the finite onedimensional oscillator. Int. J. Mod. Phys. A 18 (2003), 317-327.

[11] N. M. Atakishiyev, G. S. Pogosyan, and B. Wolf, Contraction of the finite radial oscillator. Int. J. Mod. Phys. A 18 (2003), 329-341. 
[12] N. M. Atakishiyev, L. E. Vicent, and B. Wolf, Continuous vs. discrete fractional Fourier transforms. J. Comput. Appl. Math. 107 (1999), 73-95.

[13] N. M. Atakishiyev and B. Wolf, Approximation on a finite set of points through Kravchuk functions. Revista Mexicana de Física 40 (1994), 1055-1062.

[14] N. M. Atakishiyev and B. Wolf, Fractional Fourier-Kravchuk transform. J. Opt. Soc. Amer. A 14 (1997), 1467-1477

[15] L. C. Biedenharn, The quantum group $S U_{q}(2)$ and a $q$-analogue of the boson operators. J. Phys. A. 22 (1989), L873-L878.

[16] J. L. Cardoso and R. Álvarez-Nodarse, Recurrence relations for radial wave functions for the $\mathrm{N}$-th dimensional oscillators and hydrogenlike atoms. J. Phys. A: Math. Gen. 36 (2003) 2055-2068.

[17] J.S. Dehesa and R.J. Yañez, Fundamental recurrence relations of functions of hypergeometric type and their derivatives of any order. Nuovo Cimento 109B (1994), 711-23.

[18] R. Koekoek and R. F. Swarttouw, The Askey-scheme of hypergeometric orthogonal polynomials and its $q$-analog. Reports of the Faculty of Technical Mathematics and Informatics No. 98-17. Delft University of Technology, Delft, 1998 (math.CA/9602214 at arXiv.org).

[19] M. Lorente, Raising and lowering operators, factorization method and differential/difference operators of hypergeometric type. J. Phys. A: Math. Gen. 34 (2001), 569-588.

[20] M. Lorente, Integrable systems on the lattice and orthogonal polynomials of discrete variable. J. Comput. Appl. Math. 153 (2003), 321-330.

[21] A. J. Macfarlane, On $q$-analogues of the quantum harmonic oscillator and the quantum group $S U_{q}(2)$. J. Phys. A. 22 (1989), 4581-4588.

[22] A. F. Nikiforov, S. K. Suslov, and V. B. Uvarov, Classical Orthogonal Polynomials of a Discrete Variable. Springer Series in Computational Physics. Springer-Verlag, Berlin, 1991.

[23] A.F. Nikiforov and V.B. Uvarov, Special Functions of Mathematical Physics. Birkhäuser, Basel, 1988.

[24] R.J. Yañez, J.S. Dehesa, and A.F. Nikiforov, The three-term recurrence relations and the differentiation formulas for functions of hypergeometric type. J. Math. Anal. Appl. 185 1994, 855-866.

Departamento de Análisis Matemático, Facultad de Matemática, Universidad de Sevilla. Apdo. Postal 1160, Sevilla, E-41080, Sevilla, Spain and Instituto Carlos I de Física Teórica y Computacional, Universidad de Granada. E-18071 Granada, SPAIN

E-mail address: address ran@us.es

Departamento de Matemática, Universidade de Trás-os-Montes e Alto DouRo. Apartado 202, 5001 - 911 Vila Real, Portugal

E-mail address: jluis@utad.pt 